\title{
Prolonged androgen deprivation leads to downregulation of androgen receptor and prostate-specific membrane antigen in prostate cancer cells
}

\author{
TIANCHENG LIU, LISA Y. WU, MELODY D. FULTON, JACQUELINE M. JOHNSON and CLIFFORD E. BERKMAN \\ Department of Chemistry, Washington State University, Pullman, WA 99164, USA
}

Received July 23, 2012; Accepted September 12, 2012

DOI: $10.3892 /$ ijo.2012.1649

\begin{abstract}
Emergence of androgen-independent cancer cells during androgen deprivation therapy presents a significant challenge to successful treatment outcomes in prostate cancer. Elucidating the role of androgen deprivation in the transition from an androgen-dependent to an androgen-independent state may enable the development of more effective therapeutic strategies against prostate cancer. Herein, we describe an in vitro model for assessing the effects of continuous androgendeprivation on prostate cancer cells ( $\mathrm{LNCaP}$ ) with respect to the expression of two prostate-specific markers: the androgen receptor (AR) and prostate-specific membrane antigen (PSMA). Compared with androgen-containing normal growth medium, androgen-deprived medium apparently induced the concomitant downregulation of AR and PSMA over time. Decreased protein levels were confirmed by fluorescence imaging, western blotting and enzymatic activity studies. In contrast to the current understanding of AR and PSMA in prostate cancer progression, our data demonstrated that androgen-deprivation induced a decrease in AR and PSMA levels in androgen-sensitive LNCaP cells, which may be associated with the development of more aggressive disease-state following androgen deprivation therapy.
\end{abstract}

\section{Introduction}

Prostate cancer remains the second leading cause of cancer death for Northern American men. According to the National Cancer Institute, it is estimated that there will be 241,740 new cases and 28,170 deaths from prostate cancer in the United States in 2012 (http://www.cancer.gov/cancertopics/types/prostate). Initially, prostate cancer cells depend on androgen stimulation for growth and proliferation, and sensitive to androgen deprivation therapy. Unfortunately most recurrent tumors return within two years with an androgen-independent state and an aggressive, meta-

Correspondence to: Dr Clifford E. Berkman, Department of Chemistry, Washington State University, Pullman, WA 99164-4630, USA

E-mail: cberkman@wsu.edu

Key words: androgen receptor, prostate-specific membrane antigen, androgen deprivation, prostate cancer static phenotype. As of yet there is no effective treatment for hormone-refractory prostate cancer $(1,2)$.

Androgens and the androgen receptor (AR) are critical components that govern the development, differentiation, and functionality of the prostate gland and accessory reproductive tissues, and are also involved in prostate tumorigenesis (3). These pathological functions are dependent on the androgenactivated androgen receptor, which can act as a transcription factor to regulate the expression of multiple genes. Recently, several possible mechanisms $(4,5)$ have been proposed for the progression of androgen-independent prostate cancer during androgen-deprivation therapy. Of them, mutation, amplification $(5,6)$, and expression alternative-splice variants $(7)$ of the androgen receptor are related to the adaptation of recurrent prostate cancer to low levels of androgen during androgendeprivation therapy. Androgen deprivation also induces neuroendocrine differentiation (8) of prostate cancer cells leading to autocrine/paracrine signaling pathways for survival. In addition, androgen-independent prostate cancer cells have been characterized to exhibit stem cell-like properties $(9,10)$.

Prostate-specific membrane antigen (PSMA), a type II membrane protein, has been found to be upregulated and strongly expressed on prostate tumor cells and as a consequence, it has attracted significant attention not only as a tumor marker for disease progression but also as both an imaging and therapeutic target for prostate cancer (11). Although previous studies revealed that PSMA expression was negatively regulated by androgen stimulation in AR-positive cells (12-14) and downregulation of AR expression was mediated by androgendeprivation in an established androgen-independent subline derived from the androgen-sensitive LNCaP cell line (4), there are no reports which investigate the expression of both AR and PSMA at the protein level after long-term androgen deprivation. Therefore, this study was designed to identify the effect of long-term androgen-deprived growth on these two prostate cancer markers with respect to possible implications on diagnostic and therapeutic strategies for the androgen-independent disease-state. First, androgen-sensitive LNCaP cells were cultured in androgen depleted media up to 20 passages. Second, the altered expression of PSMA and AR at the protein levels induced by androgen deprivation was determined by western blotting, fluorescence imaging analysis, and enzymatic activity. Our data suggest that long-term androgen deprivation may induce the concomitant time-dependent downregulation 
of both AR and PSMA to progress toward a more aggressive, androgen-independent prostate cancer disease state.

\section{Materials and methods}

Cell lines and reagents. The human prostate cancer cell lines LNCaP and PC-3 were obtained from the American Type Culture Collection (Manassas, VA, USA). The rabbit polyclonal anti-AR antibody (N-20) was obtained from Santa Cruz Biotechnology, Inc. (Santa Cruz, CA, USA). The goat anti-rabbit secondary antibody-FITC and the mouse monoclonal anti-GAPDH antibody (clone GAPDH-71.1) were obtained from Sigma-Aldrich (St. Louis, MO, USA). The mouse monoclonal anti-PSMA antibody 7E11 was graciously provided by Cytogen Corp. (Princeton, NJ, USA). Protein blocking solution was obtained from BioGenex (San Ramon, CA, USA). Hoechst 33342 was obtained from Invitrogen-Molecular Probes (Carlsbad, CA, USA). Cy5.5-CTT-54.2 was prepared by our lab as described previously (15). Halt Protease Inhibitor Cocktail (100X) was purchased from Thermo Fisher Scientific (Rockford, IL, USA). All other chemicals and cell-culture reagents were purchased from Fisher Scientific (Sommerville, NJ, USA) or Sigma-Aldrich.

Cell culture. $\mathrm{LNCaP}$ and PC-3 cells were grown in T-75 flasks with normal growth media [RPMI-1640 containing 10\% heatinactivated fetal calf serum (FBS), $100 \mathrm{U}$ of penicillin and $100 \mu \mathrm{g} / \mathrm{ml}$ streptomycin] in a humidified incubator at $37^{\circ} \mathrm{C}$ with $5 \% \mathrm{CO}_{2}$. Otherwise, for androgen-deprivation growth, cells were cultured with conditioned media [RPMI-1640 containing 10\% charcoal-stripped fetal bovine serum, $100 \mathrm{U}$ of penicillin and $100 \mu \mathrm{g} / \mathrm{ml}$ streptomycin]. Confluent cells were detached with a $0.25 \%$ trypsin $0.53 \mathrm{mM}$ EDTA solution, harvested, and plated in two-well slide chambers at a density of $4 \times 10^{4}$ cells/well. Cells were grown for three days before conducting the following experiments.

Immunofluorescence detection of AR. The LNCaP cells, grown under androgen deprivation condition for over time $(5,10$ and 20 passages), were cultured for 3 days on the slides in the conditioned media. For 2-day androgen-deprivation treatment, LNCaP cells were seeded on slides with normal growth media for 1-day growth, and replaced with conditioned media for another 2-day growth. Normal LNCaP cells and PC-3 cells were used for the AR-positive and AR-negative control respectively. These cells were seeded on slides with normal growth media for 3 days. Slides with 3-day growth cells in normal growth media or conditioned media were washed twice in PBS buffer (phosphate buffered saline), fixed in 4\% paraformaldehyde in PBS buffer for $15 \mathrm{~min}$ at room temperature, and permeabilized with $0.2 \%$ Triton X-100 in PBS buffer for 5 min at room temperature. The permeabilized cells were blocked in block buffer $(0.1 \%$ Tween-20,5\% goat normal serum in PBS buffer) for $2 \mathrm{~h}$ at room temperature and incubated with primary anti-AR antibody (100X diluted in block buffer) overnight at $4^{\circ} \mathrm{C}$. After washing, the cells were incubated with a secondary antibody (goat anti-rabbit IgG-FITC, 40X diluted in 1\% BSA, PBS buffer) for $2 \mathrm{~h}$ at room temperature, counterstained with Hoechst 33342, and mounted in Vectashield ${ }^{\circledR}$ Mounting Medium (Vector Laboratories, Inc., Burlingame, CA, USA) for confocal microscopy.
Affinity imaging of PSMAs with Cy5.5-CTT-54.2. The cells cultured on the 2-well slides were washed twice with warm medium $\left(37^{\circ} \mathrm{C}\right) \mathrm{A}$ (phosphate-free RPMI-1640 containing $1 \%$ FBS), then incubated with $1 \mathrm{ml}$ of Cy5.5-CTT-54.2 $(10 \mu \mathrm{M})$ in warm medium $\mathrm{A}$ for $1 \mathrm{~h}$ in a humidified incubator at $37^{\circ} \mathrm{C}$ and $5 \% \mathrm{CO}_{2}$. The above treated cells were washed three times with cold-KRB buffer pH 7.4 (mmol/l: $\mathrm{NaCl} 154.0, \mathrm{KCl} 5.0, \mathrm{CaCl}_{2}$ 2.0, $\mathrm{MgCl}_{2}$ 1.0, HEPES 5.0, D-glucose 5.0) and fixed with $4 \%$ paraformaldehyde in KRB for $15 \mathrm{~min}$ at room temperature. The cellular nuclei were counterstained with Hoechst 33342, and then mounted in Vectashield Mounting Medium for microscopy.

Confocal laser scanning microscopy. Cells were visualized under 40x oil immersion objective using an LSM 510 META laser scanning microscope. Hoechst 33342 was excited with a Diode laser (405 nm), and the emission was collected with a BP420-480-nm filter. AR immunofluorescence (with goat anti-rabbit IgG-FITC) was excited at $488 \mathrm{~nm}$ using an Argon laser, and the emission was collected with a LP505-nm filter. PSMA-targeted imaging with Cy5.5-CTT-54.2 was excited using $633 \mathrm{~nm}$ from a HeNe laser, and the emission collected with an LP 650-nm filter. To reduce interchannel crosstalk, a multi-tracking technique was used, and images were taken at a resolution of $1,024 \times 1,024$ pixels. Confocal scanning parameters were set up so that the control cells without treatment did not have background fluorescence. The imaging colors of the fluorescent dyes, Hoechst 33342 and FITC, were defined as blue and green, respectively. As the emission wavelength of the near-infrared fluorescent dye Cy5.5 was beyond visible ranges, fluorescence pseudocolor of Cy5.5 was assigned as red. The images were edited by National Institutes of Health (NIH) Image J software (http://rsb.info.nih.gov/ij) and Adobe Photoshop CS2.

Whole cell lysate extraction and western blot analysis. The controls: PC-3 and LNCaP cells (cultured in normal growth media) and $\mathrm{LNCaP}$ cells under androgen deprivation over time (2 days, 5, 10 or 20 passages) were collected by scraping, washed once in ice-cold PBS, re-suspended in 3-fold cell pellet volumes of lysis buffer (1\% NP-40, $20 \mathrm{mM}$ Tris pH 8.0, $137 \mathrm{mM} \mathrm{NaCl}, 10 \%$ glycerol $)(16,17)$ supplemented with 1X Halt Protease Inhibitor Cocktail for $15 \mathrm{~min}$ on ice, then transferred to Eppendorf tubes for centrifugation at $10,000 \mathrm{~g}$ for $15 \mathrm{~min}$ at $4^{\circ} \mathrm{C}$, the supernatant was saved as whole-cell protein extracts. Protein concentrations were determined using Non-Interfering Protein Assay (G-Biosciences, St. Louis, MO, USA). Western blotting was performed as described previously with only minor modifications $(17,18)$. In brief, detergent soluble proteins $(30 \mu \mathrm{g})$ were loaded and separated on a NuPAGE ${ }^{\mathrm{TM}} 4-12 \%$ Bis-Tris Gel (Invitrogen, Carlsbad, CA, USA) by electrophoresis for $40 \mathrm{~min}$ at a constant $200 \mathrm{~V}$ under reducing conditions, and then transferred to a $0.45 \mu \mathrm{m}$ PVDF Immobilon-P Transfer Membrane (Millipore Corp., Bedford, MA, USA) at $400 \mathrm{~mA}$ for $100 \mathrm{~min}$ in a transfer apparatus-Owl Bandit VEP-2 (Owl, Portsmouth, NH, USA) according to the manufacturer's instructions. Membranes were incubated with primary antibody at corresponding dilution overnight at $4^{\circ} \mathrm{C}$ and then with horseradish peroxidase conjugated-second antibody for $1 \mathrm{~h}$ at room temperature. The immunoreactive bands were visualized using Protein Detector 


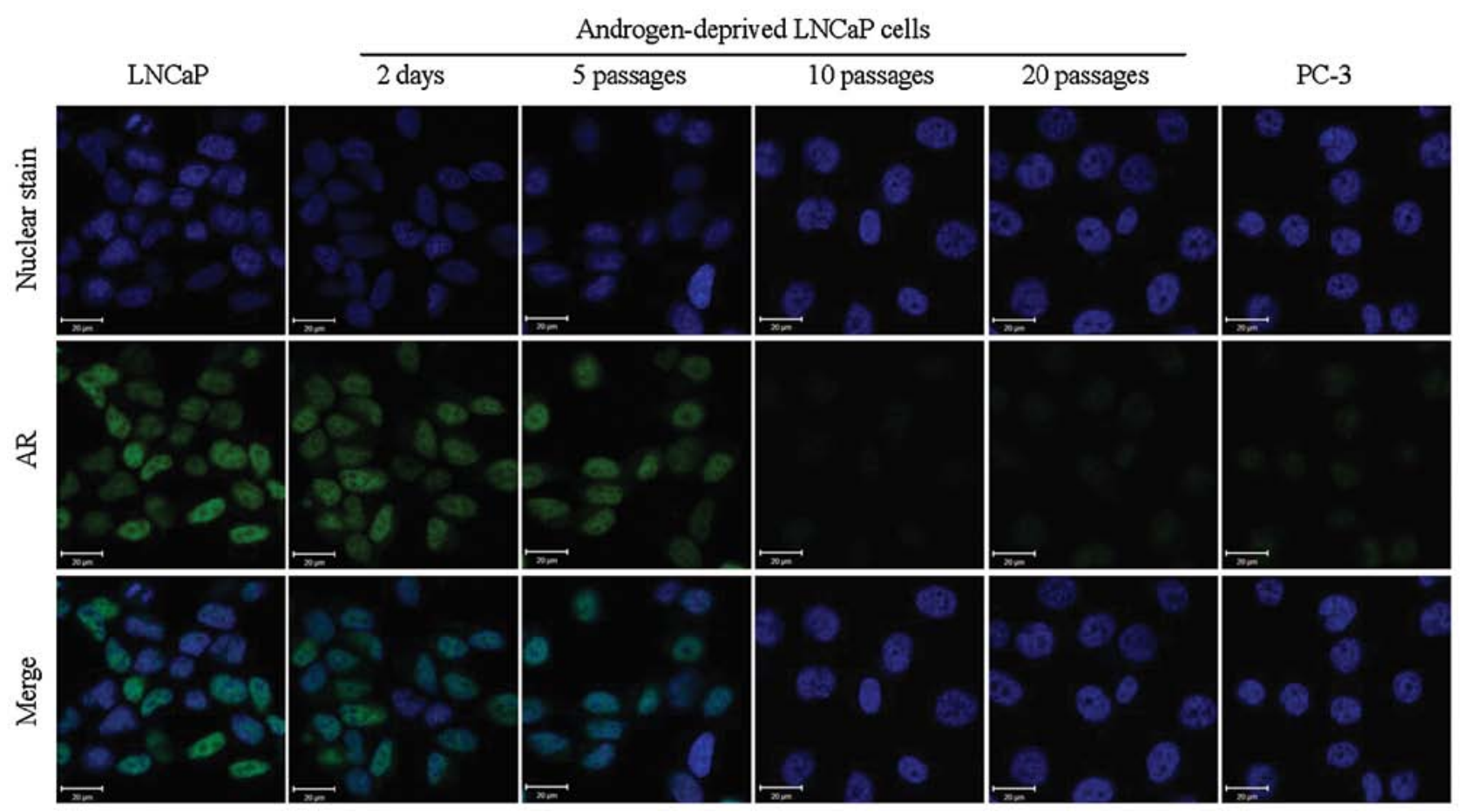

Figure 1. Downregulation of AR expression in LNCaP cells following androgen deprivation treatment. LNCaP cells, cultured in normal growth media, serve as positive controls. Strong fluorescence signals of AR were detected through the nuclei. The decreased signals were found in LNCaP cells treated with androgen deprivation for 2 days and 5 passages. There were no signals for 10 passages, 20 passages treated LNCaP cells, and AR-negative PC-3 cells. The nuclei were counterstained with Hoechst 33342 (blue). The cellular imaging was visualized by confocal microscopy; distance scale is $20 \mu \mathrm{m}$.

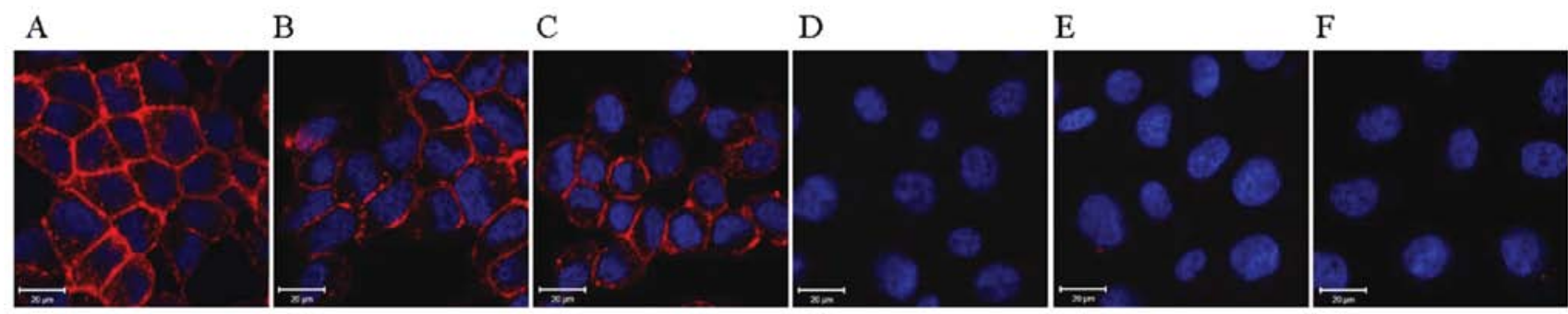

Figure 2. Downregulation of PSMA expression in androgen-deprived LNCaP cells by PSMA's affinity labeling agent Cy5.5-CTT-54.2. (A) LNCaP cells cultured in normal growth media, serve as positive controls. The following LNCaP cells were treated with androgen deprivation for 2 days (B). (C) Five passages. (D) Ten passages. (E) Twenty passages. (F) PSMA-negative PC-3 cells. The nuclei were counterstained with Hoechst 33342 . The cellular imaging was visualized by confocal microscopy; distance scale is $20 \mu \mathrm{m}$.

TMB Western Blot kit (KPL, Gaithersburg, MD, USA) following the manufacturer's instructions.

Evaluation of PSMA relative enzymatic activity. HPLC-based PSMA enzymatic activity analysis was performed in triplicate as described previously with only minor modifications $(19,20)$. Working solutions of the substrate (N-[4-(phenylazo)-benzoyl]glutamyl- $\gamma$-glutamic acid, PABGgG) were made at $10 \mu \mathrm{M}$ in Tris-buffer (50 mM, pH 7.4). Working solutions of each protein sample were diluted at $30 \mu \mathrm{g} / \mathrm{ml}$ in Tris-buffer $(50 \mathrm{mM}$, pH 7.4 containing $1 \%$ Triton X-100). A typical incubation mixture (final volume $250 \mu \mathrm{l}$ ) was prepared by the addition of $200 \mu \mathrm{l}$ Tris buffer (50 mM, pH 7.4) and PAB-Glu- $\gamma$-Glu $(25 \mu 1,10 \mu \mathrm{M})$ in a test tube. The enzymatic reaction was initiated by the addition of $25 \mu \mathrm{l}$ of the PSMA working solution. The reaction was allowed to proceed for $15 \mathrm{~min}$ with constant shaking at $37^{\circ} \mathrm{C}$ and terminated by the addition of $25 \mu \mathrm{l}$ methanolic TFA
( $2.5 \%$ trifluoroacetic acid by volume in methanol) followed by vortexing. The quenched incubation mixture was quickly buffered by the addition of $25 \mu 1 \mathrm{~K}_{2} \mathrm{HPO}_{4}(0.1 \mathrm{M})$, vortexed, iced for $15 \mathrm{~min}$, followed by centrifugation (10 $\mathrm{min}$ at 7,000 g). An $85-\mu 1$ aliquot of the resulting supernatant was subsequently quantified for the contents of substrate and product by HPLC as previously described $(21,22)$. Enzymatic activity for each protein sample was calculated from HPLC data. Activities were compared with normally-cultured LNCaP cells to evaluate relative enzymatic activity (REA).

\section{Results}

Downregulation of AR protein expression induced by androgen deprivation. In a positive control, a strong immunofluorescence signal for AR was observed throughout the nuclei of normal growth medium-cultured LNCaP cells (Fig. 1). In contrast, 


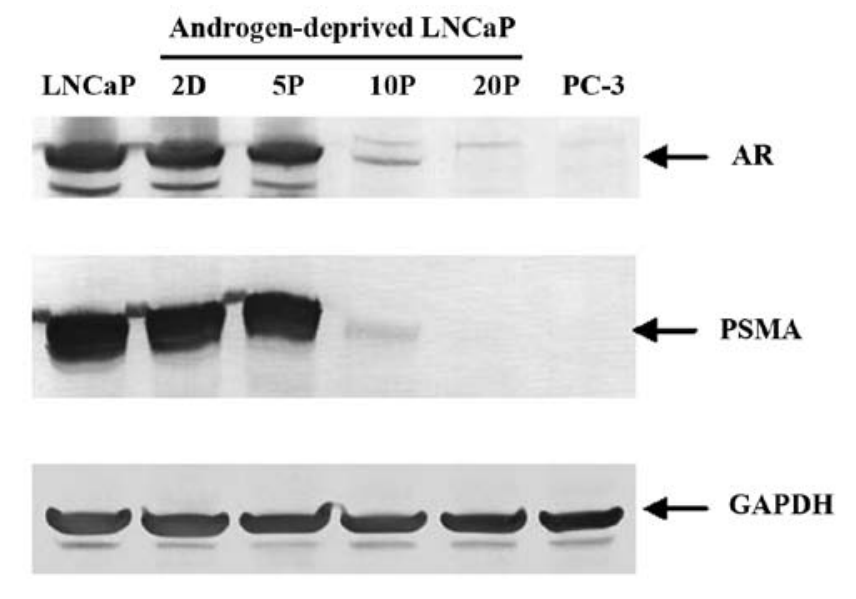

Figure 3. AR and PSMA expression were detected by western blotting. PSMA expression was downregulated in LNCaP cells treated with androgen deprivation for 2 days (2D) and 5 passages (5P), and very weak expression in cells treated for 10 passages (10P), completely undetectable in cells treated for 20 passages (20P). LNCaP cells cultured in normal growth media, serve as PSMA-positive controls, and PC-3 cells are designed as negative controls. GAPDH expression was detected to serve as a protein loading controls.

there is no signal for AR in AR-negative PC-3 cells (Fig. 1). Compared to the positive control, androgen deprivation induced downregulation of AR protein expression in LNCaP cells over time; AR was not detectable after 10 passages (Fig. 1). Based on these results, it is clear that downregulation of AR protein expression is a cell-adaptable response to prolonged androgen deprivation. Although the presented results were not consistent with the previous report which demonstrated that the administration of androgens resulted in downregulated AR mRNA levels in LNCaP cells (23), our data matched with androgenindependent LNCaP subline (4).

Affinity labeling of PSMA. Cy5.5-CTT-54.2, a specific PSMA fluorescent inhibitor $\left(\mathrm{IC}_{50}=0.55 \mathrm{nM}\right)$ was designed and evaluated for PSMA-targeted fluorescence imaging of LNCaP cells in our previous published study (15). In this study, Cy5.5-CTT-54.2 was employed to detect the change of active PSMAs on the cellular surface by fluorescence imaging of androgen-deprived LNCaP cells. Consistent with the results for AR immunofluorescence study above, the greatest cell labeling by Cy5.5-CTT-54.2 was observed for LNCaP cells cultured in normal growth media (Fig. 2A), with decreased signals through 5 passages under androgen-deprived conditions (Fig. 2B and C), and no detectable signals by 10 passages under the same conditions (Fig. 2D and E), similar to that of PSMA-negative PC-3 cells (Fig. 2F).

Western blot analysis. Western blot analysis further confirmed that the total amount of AR and PSMA decreased over time with a dramatic loss by 10 passages and absent after 20 passages in androgen-deprived conditions; GAPDH served as a protein loading controls (Fig. 3). These data suggest that downregulation of both AR and PSMA expression is dependent on the androgen levels and the length of time of androgen deprivation during cell growth.

PSMA relative enzymatic activity (REA). Analysis of PSMA relative enzymatic activity revealed that there was an

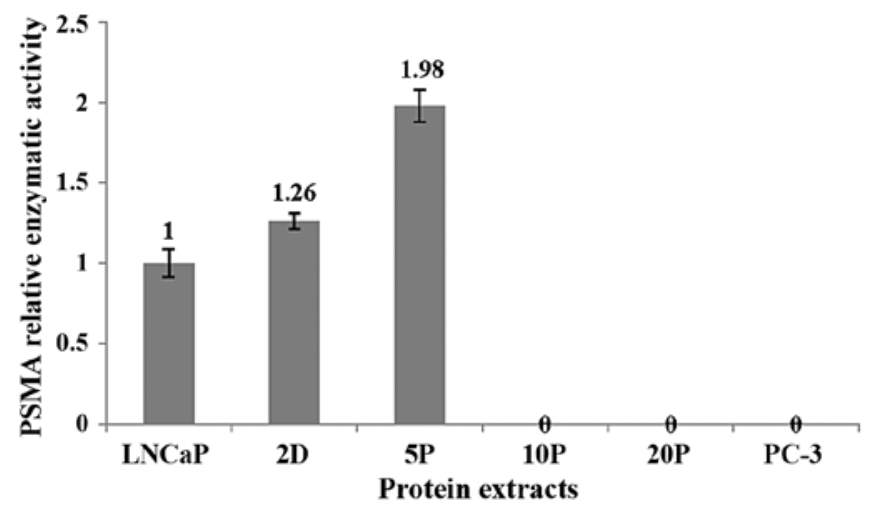

Figure 4. PSMA relative enzymatic activity (REA) increased for the protein extracts from short-term androgen deprived $\mathrm{LNCaP}$ cells: 2 days $(\mathrm{REA}=1.26)$ and 5 passages $(\mathrm{REA}=1.98)$. No detectable PSMA enzymatic activity observed for the protein extracts from long-term androgen deprived LNCaP cells (10 and 20 passages), similar to that of PSMA-negative PC-3 cells. PSMA enzymatic activity of the protein extract, from LNCaP cells cultured in normal growth media, served as a standard with a $\mathrm{REA}=1$.

apparent increase for whole-cell protein samples for 2 days $(\mathrm{REA}=1.26)$ and 5 passages $(\mathrm{REA}=1.98)$, compared to the membrane-protein sample from normally-cultured LNCaP cells (Fig. 4). However, there was no detectable PSMA enzymatic activity after 10 passages in prolonged androgendeprived conditions, similar to that of PSMA-negative PC-3 cells (Fig. 4), and consistent with the PSMA expression (Figs. 2 and 3).

\section{Discussion}

Our data suggest that long-term androgen-depletion may induce downregulation of AR and PSMA which may lead to a diagnostically and therapeutically elusive androgenindependent disease-state. These new data provide additional knowledge of androgen-independent prostate cancer progression compared to previous studies, which primarily focused on changes in AR ligand binding specificity that may result from gene structure changes (e.g., mutation, amplification, or spliced variant expression) or AR ligand-independent activation arising from alternative signal pathways that activate AR activity at the castration level of androgen $(2,24)$. With respect to PSMA, it is thought that increased expression is correlated with prostate cancer progression, especially in recurrent, metastatic cancers after androgen deprivation therapy $(25,26)$.

While increased or consistent AR levels have been reported in established androgen-independent LNCaP sublines $(27,28)$, loss of AR expression has been detected in other LNCaP sublines (4). Although all androgen-independent LNCaP sublines have been derived from a parent androgen-sensitive LNCaP cell line, they have been established in different labs under various conditions such as passage number of starting parent LNCaP cells, culture conditions, and duration of propagation under androgen-deprived growth conditions, which may result in different characteristics. In addition, the parent androgen-sensitive LNCaP cell line is not a homogeneous population, but is a multiple hypotetraploid mixture including cells with $84,86,87$ or more chromosomes (product description 
of CRL-1740, http://www.atcc.org/). Single or a combination of multiple factors may contribute to these contradictory observations on AR expression in these established $\mathrm{LNCaP}$ sublines. A recent microarray analysis on patient tissues revealed that strong AR expression was detected in benign prostate (83\%), localized prostate cancer $(100 \%)$, and lymph node metastasis $(80 \%)$, but less $(40 \%)$ in metastatic hormone-resistant prostate cancer (3). It was also noticed that two highly aggressive androgen-independent prostate cancer cell lines: DU145 and PC-3 are double-negative of AR and PSMA (29) and the loss of their AR and PSMA expression is due to epigenetic silencing by $\mathrm{CpG}$ island hypermethylation of their promoter regions (29-31). The DU145 and PC-3 lines were derived from brain and bone metastases of prostate cancer, whereas the AR(+), PSMA(+) LNCaP cell line was derived from a lymph node metastasis. The attributes of these three cell lines are consistent with the above clinical analysis for AR expression. Although the molecular basis for downregulation of AR and PSMA expression still remains unclear, the present study reveals a dynamic progression in the loss of AR and PSMA expression during androgen deprivation. We also observed that LNCaP cells gained the capability of suspension growth and more rapid proliferation after 10 passages in culture with $10 \%$ charcoal-stripped fetal bovine serum and RPMI-1640 media (data not shown). Combined, the data suggest that the protein expression levels of AR and PSMA may correlate to the progression and metastatic sites of prostate cancer. Also, highly metastatic, aggressive, and androgen-independent prostate cancer cells are more likely to exhibit an AR(-) and PSMA(-) genotype. While some evidence is suggestive of novel roles for AR and PSMA as tumor-suppressor activity in androgen-independent prostate cancer cells $(2,32)$, the loss of AR and PSMA expression may be a consequence of dedifferentiation or a stem cell-like transition of prostate cancer cells (33) induced by androgen deprivation.

It was observed that short-term androgen deprivation (up to 5 passages) lead to an apparent increase of PSMA enzymatic activity (Fig. 4) while cell labeling with a fluorescent PSMA inhibitor decreased for these time-points (Fig 2). This inconsistency may implicate a possible specific post-translational modification change (e.g., $N$-glycosylation pattern), which occurs during short-term androgen deprivation resulting in a modified PSMA with improved enzymatic activity and a relative loss of either affinity for inhibitors or cell-surface expression. In previous studies with cell lines and patient samples, different $N$-glycosylation patterns for PSMA have been detected $(34,35)$. Furthermore, it has been confirmed that the different $\mathrm{N}$-glycosylation patterns can strongly affect PSMA enzymatic activity $(34,36)$. In addition, changes in substrate specificity and enzymatic activity were exhibited by brain GCP II $(37,38)$, which shares sequence homology with PSMA (39), but is expressed in a different tissue.

In conclusion, our in vitro data suggest that the continuous, long-term androgen deprivation induces downregulation or loss of AR and PSMA expression in prostate cancer cells, which may have significance in the progression toward a more aggressive, metastatic prostate cancer disease-state during androgen deprivation therapy. The implication of these observations is that it may be critical to identify $\operatorname{AR}(+)$ or PSMA(+) tumors early to ensure the therapeutic efficacy of novel
AR- or PSMA-targeted agents to treat recurrent, metastatic, and hormone-refractory prostate cancer patients. However, an unsolved question remains whether these androgenindependent LNCaP cells originate from the minor population of stem-like cancer cells or result from the accumulation of the changes in genetic structures, gene expression profiles, or alternative signal pathways in androgen-sensitive LNCaP cells.

\section{Acknowledgements}

The authors thank Cytogen Corp. (Princeton, NJ, USA) for the gift of the mouse monoclonal antibody 7E11, and extend their gratitude for technical assistance from C. Davitt and V. LynchHolm at the WSU Franceschi Microscopy and Imaging Center. This study was supported in part by the National Institutes of Health (R01CA140617).

\section{References}

1. Gustavsson H, Welen K and Damber JE: Transition of an androgen-dependent human prostate cancer cell line into an androgen-independent subline is associated with increased angiogenesis. Prostate 62: 364-373, 2005.

2. Niu Y, Altuwaijri S, Lai KP, et al: Androgen receptor is a tumor suppressor and proliferator in prostate cancer. Proc Natl Acad Sci USA 105: 12182-12187, 2008.

3. Davis JN, Wojno KJ, Daignault S, et al: Elevated E2F1 inhibits transcription of the androgen receptor in metastatic hormoneresistant prostate cancer. Cancer Res 66: 11897-11906, 2006.

4. Lee SO, Dutt SS, Nadiminty N, Pinder E, Liao H and Gao AC: Development of an androgen-deprivation induced and androgen suppressed human prostate cancer cell line. Prostate 67: 1293-1300, 2007.

5. Saraon P, Jarvi K and Diamandis EP: Molecular alterations during progression of prostate cancer to androgen independence. Clin Chem 57: 1366-1375, 2011.

6. Devlin HL and Mudryj M: Progression of prostate cancer: multiple pathways to androgen independence. Cancer Lett 274: 177-186, 2009.

7. Watson PA, Chen YF, Balbas MD, et al: Constitutively active androgen receptor splice variants expressed in castration-resistant prostate cancer require full-length androgen receptor. Proc Natl Acad Sci USA 107: 16759-16765, 2010.

8. Yuan TC, Veeramani S, Lin FF, et al: Androgen deprivation induces human prostate epithelial neuroendocrine differentiation of androgen-sensitive LNCaP cells. Endocr Relat Cancer 13: 151-167, 2006.

9. Zhang K and Waxman DJ: PC3 prostate tumor-initiating cells with molecular profile FAM65Bhigh/MFI2low/LEF1low increase tumor angiogenesis. Mol Cancer 9: 319, 2010.

10. Bae KM, Su Z, Frye C, et al: Expression of pluripotent stem cell reprogramming factors by prostate tumor initiating cells. J Urol 183: 2045-2053, 2010.

11. Rajasekaran AK, Anilkumar G and Christiansen JJ: Is prostatespecific membrane antigen a multifunctional protein? Am J Physiol Cell Physiol 288: C975-C981, 2005.

12. Lee SJ, Lee K, Yang X, et al: NFATc1 with AP-3 site binding specificity mediates gene expression of prostate-specificmembrane-antigen. J Mol Biol 330: 749-760, 2003.

13. Ghosh A and Heston WD: Tumor target prostate specific membrane antigen (PSMA) and its regulation in prostate cancer. J Cell Biochem 91: 528-539, 2004.

14. Israeli RS, Powell CT, Corr JG, Fair WR and Heston WD: Expression of the prostate-specific membrane antigen. Cancer Res 54: 1807-1811, 1994.

15. Liu T, Wu LY, Hopkins MR, Choi JK and Berkman CE: A targeted low molecular weight near-infrared fluorescent probe for prostate cancer. Bioorg Med Chem Lett 20: 7124-7126, 2010.

16. Matroule JY, Carthy CM, Granville DJ, Jolois O, Hunt DW and Piette J: Mechanism of colon cancer cell apoptosis mediated by pyropheophorbide-a methylester photosensitization. Oncogene 20: 4070-4084, 2001. 
17. Liu T, Wu LY and Berkman CE: Prostate-specific membrane antigen-targeted photodynamic therapy induces rapid cytoskeletal disruption. Cancer Lett 296: 106-112, 2010.

18. Liu T, Toriyabe Y and Berkman CE: Purification of prostatespecific membrane antigen using conformational epitope-specific antibody-affinity chromatography. Protein Expr Purif 49: 251-255, 2006.

19. Liu T, Wu LY, Kazak M and Berkman CE: Cell-surface labeling and internalization by a fluorescent inhibitor of prostate-specific membrane antigen. Prostate 68: 955-964, 2008.

20. Wu LY, Anderson MO, Toriyabe Y, et al: The molecular pruning of a phosphoramidate peptidomimetic inhibitor of prostatespecific membrane antigen. Bioorg Med Chem 15: 7434-7443, 2007.

21. Anderson MO, Wu LY, Santiago NM, et al: Substrate specificity of prostate-specific membrane antigen. Bioorg Med Chem 15: 6678-6686, 2007.

22. Maung J, Mallari JP, Girtsman TA, et al: Probing for a hydrophobic a binding register in prostate-specific membrane antigen with phenylalkylphosphonamidates. Bioorg Med Chem 12: 4969-4979, 2004.

23. Wolf DA, Herzinger T, Hermeking H, Blaschke D and Horz W: Transcriptional and posttranscriptional regulation of human androgen receptor expression by androgen. Mol Endocrinol 7: 924-936, 1993 .

24. Niu Y, Chang TM, Yeh S, Ma WL, Wang YZ and Chang C: Differential androgen receptor signals in different cells explain why androgen-deprivation therapy of prostate cancer fails. Oncogene 29: 3593-3604, 2010

25. Ross JS, Sheehan CE, Fisher HA, et al: Correlation of primary tumor prostate-specific membrane antigen expression with disease recurrence in prostate cancer. Clin Cancer Res 9: 6357-6362, 2003.

26. Perner S, Hofer MD, Kim R, et al: Prostate-specific membrane antigen expression as a predictor of prostate cancer progression. Hum Pathol 38: 696-701, 2007.

27. Kokontis JM, Hay N and Liao S: Progression of LNCaP prostate tumor cells during androgen deprivation: hormone-independent growth, repression of proliferation by androgen, and role for p27 $7^{\text {Kipl }}$ in androgen-induced cell cycle arrest. Mol Endocrinol 12: 941-953, 1998
28. Ishikura N, Kawata H, Nishimoto A, Nakamura R, Ishii $\mathrm{N}$ and Aoki Y: Establishment and characterization of an androgen receptor-dependent, androgen-independent human prostate cancer cell line, LNCaP-CS10. Prostate 70: 457-466, 2010.

29. Suzuki H, Ueda T, Ichikawa T and Ito H: Androgen receptor involvement in the progression of prostate cancer. Endocr Relat Cancer 10: 209-216, 2003.

30. Chlenski A, Nakashiro K, Ketels KV, Korovaitseva GI and Oyasu R: Androgen receptor expression in androgen-independent prostate cancer cell lines. Prostate 47: 66-75, 2001.

31. Mhawech-Fauceglia P, Smiraglia DJ, Bshara W, et al: Prostatespecific membrane antigen expression is a potential prognostic marker in endometrial adenocarcinoma. Cancer Epidemiol Biomarkers Prev 17: 571-577, 2008.

32. Ghosh A, Wang X, Klein E and Heston WD: Novel role of prostate-specific membrane antigen in suppressing prostate cancer invasiveness. Cancer Res 65: 727-731, 2005.

33. Tang Y, Hamburger AW, Wang L, Khan MA and Hussain A Androgen deprivation and stem cell markers in prostate cancers. Int J Clin Exp Pathol 3: 128-138, 2009.

34. Ghosh A and Heston WD: Effect of carbohydrate moieties on the folate hydrolysis activity of the prostate specific membrane antigen. Prostate 57: 140-151, 2003.

35. Holmes EH, Greene TG, Tino WT, et al: Analysis of glycosylation of prostate-specific membrane antigen derived from $\mathrm{LNCaP}$ cells, prostatic carcinoma tumors, and serum from prostate cancer patients. Prostate (Suppl) 7: 25-29, 1996.

36. Barinka C, Sacha P, Sklenar J, et al: Identification of the $\mathrm{N}$-glycosylation sites on glutamate carboxypeptidase II necessary for proteolytic activity. Protein Sci 13: 1627-1635, 2004.

37. Luthi-Carter R, Barczak AK, Speno H and Coyle JT: Hydrolysis of the neuropeptide $\mathrm{N}$-acetylaspartylglutamate (NAAG) by cloned human glutamate carboxypeptidase II. Brain Res 795: 341-348, 1998.

38. Tiffany CW, Lapidus RG, Merion A, Calvin DC and Slusher BS: Characterization of the enzymatic activity of PSM: comparison with brain NAALADase. Prostate 39: 28-35, 1999.

39. Luthi-Carter R, Barczak AK, Speno H and Coyle JT: Molecular characterization of human brain $\mathrm{N}$-acetylated alpha-linked acidic dipeptidase (NAALADase). J Pharmacol Exp Ther 286: 1020-1025, 1998. 